\title{
Diferentes substratos e ambientes no enraizamento in vitro de mirtilo
}

\author{
Different substrates and environment conditions on in vitro rooting of blueberry
}

\author{
Cláudia Roberta Damiani ${ }^{\mathrm{I}}$ Márcia Wulff Schuch ${ }^{\mathrm{II}}$
}

\begin{abstract}
O objetivo neste trabalho foi comparar a capacidade de enraizamento in vitro de mirtilo (Vaccinium ashei Reade cv. 'Delite' e V. corymbosum L. cv. 'Georgiagem') e estudar o efeito de substratos alternativos ao ágar (perlita, vermiculita e ágar + carvão ativado) adicionados ao meio de cultura ' $W P M$ ' contendo $7 \mu M L^{-1}$ de $A I B$ e o efeito da luminosidade por meio do cultivo em diferentes ambientes: sala de crescimento convencional e casa de vegetação. Concluiu-se que o uso de perlita, seguido da vermiculita, promovem o aumento do número e comprimento de raízes, matéria fresca total e radicular e maiores porcentagens de enraizamento. A adição de carvão ativado inibe o enraizamento. $O$ ambiente de cultivo não alterou o número de raizes formadas. A cultivar 'Georgiagem' apresentou maiores porcentagens de enraizamento quando comparada a cv. 'Delite', independentemente do local de cultivo e substrato.
\end{abstract}

Palavras-chave: Vaccinium ashei, V. corymbosum, luz natural, carvão ativado, perlita, vermiculita.

\section{ABSTRACT}

The aim of this research was to compare the in vitro rooting ability of blueberry (Vaccinium ashei Reade $c v$. 'Delite' and V. corymbosum L. cv. 'Georgiagem'), to study the effect of alternative substrate instead of agar (perlite, vermiculite and agar + activated charcoal) added to the WPM culture medium containing $7 \mu M L^{-1}$ of $A I B$, and the light effect by raising the explants in different growth environment: conventional growth room and greenhouse. The use of perlite followed by vermiculite increased roots number and length, total fresh weight and root weight and rooting percentage. The addition of activated charcoal inhibited rooting. The environment condition did not alter the number of formed roots. The cv. 'Georgiagem' showed the highest percentages of rooting whether compared cv. 'Delite', independently of growth environment and substrate.

Key words: Vaccinium ashei, V. corymbosum, natural light, activated charcoal, perlite, vermiculite.

O mirtilo, pertencente à família Ericaceae e ao gênero Vaccinium, é uma espécie frutífera de clima temperado (SHARPE, 1980), e umas das culturas mais promissoras para a Região Sul do Brasil, devido às condições edafoclimáticas favoráveis à adaptação de muitas cultivares. No entanto, uma das limitações do seu cultivo é a baixa disponibilidade de mudas. O mirtilo é normalmente propagado por estaquia (HOFFMANN et al., 1994), porém a baixa produção de ramos para a produção de estacas e a dificuldade de enraizamento de algumas cultivares têm limitado sua propagação. Uma alternativa para aumentar a disponibilidade de mudas dessa espécie é o uso da micropropagação in vitro.

A micropropagação in vitro apresenta regime heterotrófico, com reduzida troca gasosa e baixa intensidade luminosa, o que pode produzir plantas com um elevado conteúdo de água, com grande risco de desidratação e morte durante a aclimatização (Kubota \& Kozai, 1992).

Para corrigir as desordens fisiológicas, provocadas pela baixa intensidade luminosa e facilitar as trocas gasosas no meio, uma alternativa viável é a

'Laboratório de Micropropagação de Plantas Frutíferas, Universidade Federal de Pelotas (UFPel), Campus Capão do Leão, Pelotas, RS, Brasil. E-mail: claudami2004@yahoo.com.br. Autor para correspondência.

IIDepartamento de Fitotecnia, Faculdade de Agronomia Eliseu Maciel (FAEM), UFPel, Pelotas, RS, Brasil. 
substituição da luz artificial pela natural e do agente geleificante (ágar ou Gelrite ${ }^{\circledR}$ ) por fibras ou materiais de suporte aerados ou porosos, como a vermiculita ou as fibras de celulose que, sob condições de menor umidade relativa, alta concentração de $\mathrm{CO}_{2}$ e alta luminosidade (observadas na micropropagação fotoautotrófica) têm favorecido o crescimento de plantas in vitro e aumentado, principalmente, seu enraizamento e a formação de raízes secundárias e de um sistema vascular normal (KOZAI \& KUBOTA, 2001), devido ao aumento da oxigenação e da disponibilidade de nutrientes na zona radicular (KOZAI \& NGUYEN; 2003).

Dentre os fatores ambientais que podem influenciar o enraizamento, destacam-se a temperatura, a luz e a umidade. O excesso de luminosidade pode ativar a enzima AIA-oxidase e esta degradar as auxinas, reduzindo assim a capacidade de enraizamento. Uma das formas de contornar esse problema é a adição de carvão ativado ao meio de cultura, que apresenta propriedades físico-químicas, que podem reduzir a intensidade de luz na região de formação de raízes e, devido a sua capacidade de adsorção, modificar a composição dos meios de cultura, adsorvendo substâncias promotoras de enraizamento e também substâncias tóxicas, fenóis e/ou quinonas, produzidas durante a autoclavagem ou liberadas de explantes, cujos tecidos sofreram injúrias (ASSIS \& TEIXEIRA, 1998).

O desenvolvimento de um sistema de enraizamento mais eficiente resulta em mudas com maior qualidade fisiológica e diminuição de perdas durante a fase de aclimatização. O objetivo no presente trabalho foi avaliar o efeito de substratos alternativos ao ágar no enraizamento in vitro de duas cultivares de mirtilo, 'Delite' e 'Gergiagem', e também determinar o melhor ambiente de cultivo.

O trabalho foi desenvolvido no Laboratório de Micropropagação de Plantas Frutíferas e em casa de vegetação do departamento de Fitotecnia, FAEM/ UFPel, Pelotas, RS. Segmentos caulinares de mirtilo, cultivados in vitro, com seis gemas, ápice caulinar e folhas foram utilizados como explantes. O delineamento experimental utilizado foi inteiramente casualizado, com fatorial $3 \times 2 \times 2$, sendo quatro repetições por tratamento. Cada repetição constituiu-se de um frasco com cinco explantes. Os tratamentos consistiram de três substratos: perlita expandida pac $>3 \mathrm{~mm}\left(200 \mathrm{mg} \mathrm{L}^{-1}\right)$, vermiculita com granulometria média $\left(200 \mathrm{mg} \mathrm{L}^{-1}\right)$ e ágar $\left(6 \mathrm{~g} \mathrm{~L}^{-1}\right)+$ carvão ativado - CA (1\%), adicionados ao meio de cultura; dois ambientes de cultivo: sala de crescimento (16 horas de fotoperíodo, densidade de fluxo de fótons no período de luz de $42 \mu \mathrm{mol} \mathrm{m}^{-2} \mathrm{~s}^{-1} \mathrm{e}$ temperatura de $25 \pm 2^{\circ} \mathrm{C}$ ) e casa de vegetação (luz natural e temperatura de $25 \pm 2^{\circ} \mathrm{C}$ ) e duas cultivares de mirtilo: 'Delite' (Vaccinium ashei Reade) e 'Georgiagem' ( $\boldsymbol{V}$. corymbosum L.).

O meio de cultura empregado foi WPM (Wood Plant Media - Lloyd \& McCown, 1980), adicionado de mio-inositol $\left(100 \mathrm{mg} \mathrm{L}^{-1}\right)$, sacarose $(30 \mathrm{~g}$ $\left.\mathrm{L}^{-1}\right)$, ácido indolbutírico - AIB $\left(7 \mu \mathrm{M} \mathrm{L}^{-1}\right)$ e substrato conforme tratamento. $\mathrm{O} \mathrm{pH}$ foi ajustado para 5,0 antes da adição dos substratos (DAMIANI \& SCHUCH, 2008). O meio de cultura foi autoclavado a $120^{\circ} \mathrm{C}$ e 1,5atm, por 20 minutos e distribuídos em Erlenmeyers com capacidade de $250 \mathrm{~mL}$, contendo $50 \mathrm{~mL}$ de meio cada.

Aos 60 dias de cultivo, foram avaliados o número de raízes; comprimento da maior raiz, porcentagem de enraizamento, matéria fresca total e matéria fresca do sistema radicular. O número de raízes foi transformado em raiz quadrada $(\mathrm{X}+0,5)$ e a porcentagem de enraizamento em arcoseno(X/100). Após a análise de variância, as médias foram comparadas pelo teste de Duncan $(\mathrm{P} \leq 0,05)$.

$\mathrm{O}$ uso de substratos alternativos ao ágar para ambas as cultivares de mirtilo, atuam positivamente no enraizamento em todas as variáveis analisadas (Tabela 1). A adição de perlita e vermiculita no meio de cultura promoveram o aumento do número e comprimento de raízes, da matéria fresca total e radicular, além de maiores porcentagens de enraizamento. Os explantes crescidos em ágar + CA apresentaram um baixo índice de enraizamento. Os resultados obtidos sugerem que o carvão ativado inibe o processo de enraizamento em mirtilo, em função de sua elevada capacidade de adsorção, modifica a composição do meio de cultura e, principalmente, inativa a ação da auxina (ASSIS \& TEIXEIRA, 1998). Estudos realizados em Arbutus xalapensis, uma espécie ornamental também pertencente à família Ericaceae, a qual difere de espécies de mirtilo, como V. corymbosum e V.ashei por desenvolver-se em meios de proliferação com $\mathrm{pH}$ mais elevado $(5,7-5,8)$, demonstraram que a adição de carvão ativado ao meio de cultura (WPM) durante a fase de enraizamento promove a ramificação e alongamento das raízes, mas inibe a formação das mesmas (MACKAY, 1996).

Para o comprimento de raiz (Tabela 2), observou-se interação significativa entre substrato e ambiente de cultivo. Em ambos os ambientes de cultivo, o uso de perlita favoreceu o crescimento e alongamento das raízes, observando-se o valor médio entre os dois ambientes de 2,09cm (Tabela 1), o que, comparado aos demais substratos, difere em cerca de $56 \%$ para a vermiculita e $83 \%$ para ágar + CA. Esse resultado pode 
Tabela 1 - Médias do número de raízes, comprimento da maior raiz, matéria fresca total, matéria fresca do sistema radicular e porcentagem de enraizamento in vitro de mirtilo, cultivares 'Georgiagem' e 'Delite', aos 60 dias de cultivo em diferentes substratos. UFPEL, Pelotas-RS, 2007.

\begin{tabular}{lccccc}
\hline & Número de raízes & $\begin{array}{c}\text { Comprimento maior } \\
\text { raiz }(\mathrm{cm})\end{array}$ & $\begin{array}{c}\text { Matéria fresca total } \\
(\mathrm{mg})\end{array}$ & $\begin{array}{c}\text { Matéria fresca do } \\
\text { sistema radicular (mg) }\end{array}$ & $\begin{array}{c}\text { Porcentagem de } \\
\text { enraizamento }\end{array}$ \\
\hline Perlita & $2,67 \mathrm{a}^{*}$ & $2,09 \mathrm{a}$ & $210,89 \mathrm{a}$ & $154,31 \mathrm{a}$ & $77,5 \mathrm{a}$ \\
Vermiculita & $1,98 \mathrm{a}$ & $0,91 \mathrm{~b}$ & $190,69 \mathrm{a}$ & $140,46 \mathrm{a}$ & $58,75 \mathrm{~b}$ \\
Ágar + CA & $0,42 \mathrm{~b}$ & $0,36 \mathrm{c}$ & $38,96 \mathrm{~b}$ & $2,56 \quad \mathrm{~b}$ & 7,5 \\
$\mathrm{CV}(\%)$ & 25,67 & 68,35 & 35,91 & 57,97 & 52,8 \\
\hline
\end{tabular}

*Valores seguidos por letras distintas na coluna diferem entre si pelo teste de Duncan $(\mathrm{P}<0,05)$. CA= Carvão ativado.

ser atribuído à característica de elevado grau de porosidade da perlita, evitando a compactação do substrato e menor resistência ao desenvolvimento radicular. Verificou-se também que os explantes enraizados em meio contendo perlita, apresentaram menor comprimento radicular sob luminosidade natural (casa de vegetação), enquanto os valores obtidos de explantes enraizados em vermiculita e em ágar + CA não diferiram estatisticamente (Tabela 2).

$\mathrm{O}$ efeito do substrato na qualidade das raízes está relacionado, principalmente, com a porosidade, a qual afeta o teor de água retido e o seu equilíbrio com a aeração (HOFFMANN et al., 1994). O uso de substratos inertes, como perlita e vermiculita também podem auxiliar na redução dos custos com o ágar (GRATTAPAGLIA\& MACHADO, 1998).

O ambiente de cultivo alterou a quantidade de matéria fresca total (dados não apresentados). Explantes enraizados na sala de crescimento apresentaram maior matéria fresca $(162,5 \mathrm{mg}$ por explante), quando comparados aos enraizados em casa de vegetação $(131,2 \mathrm{mg})$. Os dados obtidos da matéria fresca do sistema radicular, em função do ambiente de cultivo, não apresentaram diferenças significativas. No entanto, é importante salientar que explantes enraizados na sala de crescimento apresentaram maior formação de calos, principalmente, em vermiculita. $\mathrm{O}$ aumento da matéria fresca total, nas plantas enraizadas em sala de crescimento, pode estar relacionados à massa dos calos e não propriamente ao melhor crescimento e desenvolvimento.

A porcentagem de enraizamento (Tabela 2), é dependente do substrato, variando de zero a $100 \%$, no caso de explantes enraizados em ágar $+\mathrm{CA}$ e perlita, bem como também é dependente da capacidade de enraizamento da cultivar, pois, sob as mesmas condições de cultivo, 'Delite' apresentou menor porcentagem de enraizamento do que 'Georgiagem'. A propagação de mirtilo por estacas também apresentou resultados variáveis, conforme a espécie e a cultivar (HOFFMANN, 2005).

Além das características físicas, alguns atributos químicos dos substratos podem influenciar na formação e desenvolvimento inicial das plantas, tais como o pH (BASTOS et al., 2007). Para o sucesso da cultura do mirtilo, o pH do solo deve ser ácido $(4,0 \mathrm{a}$

Tabela 2 - Médias do comprimento da maior raiz durante o enraizamento e da porcentagem de enraizamento in vitro de mirtilo, cultivares 'Georgiagem' e 'Delite', aos 60 dias de cultivo, em diferentes substratos e ambientes de cultivo. UF PEL, Pelota s-RS, 2007.

\begin{tabular}{|c|c|c|c|c|c|c|c|c|}
\hline & \multicolumn{2}{|c|}{ Comprimento maior raiz $(\mathrm{cm})$} & \multicolumn{6}{|c|}{ 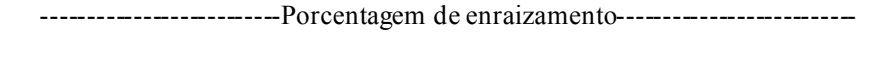 } \\
\hline & & & \multicolumn{3}{|c|}{--_-_-_Georgiagem--_-_-_-_- } & \multicolumn{3}{|c|}{ 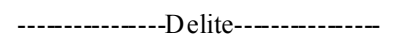 } \\
\hline & $\mathrm{SC}$ & $\mathrm{CV}$ & $\mathrm{SC}$ & $\mathrm{CV}$ & Média & $\mathrm{SC}$ & $\mathrm{CV}$ & Média \\
\hline Perlita & $2,73 \mathrm{a} \mathrm{A}^{*}$ & 1,45 a B & $100 \mathrm{a} \mathrm{A}^{* *}$ & 95 a A & $97,5^{\text {a A }}$ & 80 a A & 35 a B & $57,5^{\mathrm{aB}}$ \\
\hline Vermiculita & $0,86 \mathrm{~b} \mathrm{~A}$ & $0,97 \mathrm{ab} \mathrm{A}$ & 90 a $\mathrm{A}$ & 80 a $\mathrm{A}$ & $85^{\text {a A }}$ & $20 \mathrm{~b} \mathrm{~A}$ & 45 a A & 32,5 b в \\
\hline Ágar + CA & $0,19 \mathrm{~b} \mathrm{~A}$ & $0,53 \mathrm{~b} \mathrm{~A}$ & $20 \mathrm{~b} \mathrm{~A}$ & $10 \mathrm{~b} \mathrm{~A}$ & $15^{\text {a A }}$ & $0 \mathrm{~b} \mathrm{~A}$ & $0 \mathrm{~b} \mathrm{~A}$ & $0^{\mathrm{cA}}$ \\
\hline $\mathrm{CV}(\%)$ & \multicolumn{2}{|c|}{68,35} & \multicolumn{6}{|c|}{52,8} \\
\hline
\end{tabular}

*Valores do comprimento de raiz seguidos por letras distintas (minúsculas na coluna e maiúsculas na linha) diferem entre si pelo teste de Duncan $(\mathrm{P}<0,05)$. CA= carvão ativado.

**Valores da porcentagem de enraizamento seguidos por letras distintas (minúsculas na coluna e maiúsculas na linha) comparam substratos e ambientes dentro da cultivar e letras distintas sobrescritas comparam as cultivares em função dos substratos (médias).

Ciência Rural, v.39, n.2, mar-abr, 2009. 
5,2), deve conter elevado teor de matéria orgânica (superior a 5\%) e uma boa retenção de umidade e boa drenagem (HOFFMANN, 2005). O elevado índice de enraizamento e de desenvolvimento da parte aérea, obtidos neste experimento, utilizando perlita, podem ser atribuídos ao fato desse substrato ser quimicamente inerte, com pH neutro e retenção iônica desprezível e, dessa forma, não alterar a composição do meio de cultura, bem como suas propriedades químicas, como $\mathrm{pH}$.

Concluiu-se que a adição de carvão ativado inibe o enraizamento in vitro de mirtilo, enquanto a perlita promove o desenvolvimento do sistema radicular e aumenta a porcentagem de enraizamento.

O enraizamento in vitro de mirtilo pode ser realizado em casa de vegetação, sob luminosidade natural, utilizando perlita ou vermiculita como substrato.

\section{REFERÊNCIAS}

ASSIS, T.F.; TEIXEIRA, S.L. Enraizamento de plantas lenhosas. In: TORRES, A.C. et al. Cultura de tecidos e transformação genética de plantas. Brasília: Embrapa - SPI / EmbrapaCNPH, 1998. V.1, p.183-260.

BASTOS, D.C. et al. Diferentes substratos na produção de porta-enxertos de caramboleira. Ciência e Agrotecnologia, Lavras, v.31, n.2, p.312-316, 2007.

DAMIANI, C.R.; SCHUCH, M.W. Multiplicação fotoautotrófica de mirtilo através do uso de luz natural. Revista Brasileira de Fruticultura, Jaboticabal, v.30, n.2, p.000000, 2008 (no prelo).

GRATTAPAGLIA, D.; MACHADO, M.A. Micropropagação. In: TORRES, A.C. et al. Cultura de tecidos e transformação genética de plantas. Brasília: Embrapa-SPI / Embrapa-CNPH, 1998. V.1, p.183-260.

HOFFMANN, A. Mirtilo. Aspectos gerais da cultura. Acesso em 15 de agosto de 2005. Online. Disponível na internet: http:/www.cnpv.embrapa.br/unidade/rh/pesquisadores. html\#Hoffmann.

HOFFMANN, A. Propagação de mirtilo (Vaccinium ashei Reade) através de estacas. 1994. 94f. Dissertação (Mestrado em Agronomia) - Faculdade de Agronomia Eliseu Maciel, Universidade Federal de Pelotas, Pelotas, RS.

KOZAI, T.; NGUYEN, Q.T. Photoautotrophic micropropagation of woody and tropical plants. In: JAIN, S.M.; ISHII, K. Micropropagation of woody trees and fruits. Dordrecht: Kluwer Academic, 2003. p.757-781.

KOZAI, T; KUBOTA, C. Developing a photoautotrophic micropropagation system for woody plants. Journal of Plant Research, Tokyo, v.114, p.525-537, 2001.

KUBOTA, C.; KOZAI, T. Growth and net photosynthetic rate of Solanum tuberosum in vitro under forced and natural ventilation. HortScience, Alexandria, v.27, p.1312-1314, 1992.

LLOYD, G.; McCOWN, B. Commercially feasible micropropagation of mountain laurel (Kalmia latifolia) by use of shoot-tip culture. Proceedings of the International Plant Propagation Society, Seattle, v.30, p.421-427, 1980.

MACKAY, W.A. Micropropagation of Texas madrone, Arbutus xalapensis H.B.K. Horticulturae Science, Alexandria, v.31, n.6, p.1028-1029, 1996.

SHARPE, R.H. Consultants report. Pelotas: IICA/ EMBRAPA - UEPAE, 1980. 11p. 